\title{
Self-Stabilization and Behavioral Diversity of Embodied Adaptive Locomotion
}

\author{
Fumiya Iida and Rolf Pfeifer \\ Artificial Intelligence Laboratory, \\ Department of Informatics, University of Zurich, \\ Andreasstrasse 15, 8050 Zurich, Switzerland \\ [iida,pfeifer] @ifi.unizh.ch, \\ http://www.ifi.unizh.ch/ailab/
}

\begin{abstract}
Locomotion is of fundamental importance in understanding adaptive behavior. In this paper we present two case studies of robot locomotion that demonstrate how higher level of behavioral diversity can be achieved while observing the principle of cheap design. More precisely, it is shown that, by exploiting the dynamics of the system-environment interaction, very simple controllers can be designed which is essential to achieve rapid locomotion. Special consideration must be given to the choice of body materials. We conclude with some speculation about the importance of locomotion for understanding cognition.
\end{abstract}

\section{Introduction}

Normally, when dealing with locomotion, the focus is on the control aspects, as is illustrated by most of the research in the field of robotics(e.g. [1-6]). With a few notable exceptions, the physical body dynamics has not been taken into account, and has not been sufficiently exploited. As a result, most approaches still suffer from being relatively slow and lack a high degree of adaptability because of the enormous real-time computational requirements.

The idea of exploiting dynamics was introduced by the studies of Passive Dynamic Walkers [7-9], which demonstrated that given certain environmental conditions and a proper morphological design of the robot, biped walking is possible without, or with very little, computation and actuation. Because the Passive Dynamic Walker exploits the specific interaction with the environment to an extreme extent, its ecological niche is very narrow: it can only walk down a slope with a particular angle of inclination, and the friction coefficients must be within a small range. The exploitation of the specifics of the ecological niche always entails trade-offs: if the conditions do not hold any more, for example, if the angle of inclination is changed, the Passive Dynamic Walker will fall over.

Previously, a set of design principles of autonomous agents have been proposed [10]. In this framework, the concept of cheap design states that good designs exploit the physics of the system-environment interaction and the constraints of the ecological niche, which substantially reduces the complexity of 
the control architecture. The mechanisms underlying adaptive behavior or intelligence in general, therefore, cannot be reduced to some kind of internal representation. Rather, it is the interplay between the neural system and the "hardware" of the body that constitutes these mechanisms. So far, the interaction between body and control dynamics of locomotion has been only partially explored (e.g. [11-13]), and the design principles of such a mobile robot are not fully understood. Generally, cheap design, implies trade-offs which reduces the adaptability to environmental changes, because the system is relying on the environmental constraints. However, if interpreted properly as the exploitation of constraints, "cheap design" can be applied to more complex behaviors.

Based on biomechanical studies, the legged systems have been investigated, which explained the elastic components in the legs can provide the property of self-stabilization during locomotion process [14-18]. An interesting aspect of this approach is that the cheap design (i.e. having passive elasticity in the body) is employed not only for relaxing the control duty, but also to achieve the energy efficient and rapid locomotion.

To better understand the nature of cheap design, in this paper, we investigate the use of body dynamics with two case studies of locomotion robots, called "Stumpy" and "Puppy". We will attempt to extract the design principles for achieving behavioral diversity, which is a prerequisite for an adaptive robot. As shown in the experimental results below, a robot which properly exploits its intrinsic body dynamics and self-stabilization mechanisms, is able to display a high level of behavior diversity. We start by describing the design, the control, and the various gaits of the hopping robot "Stumpy". Then we introduce the quadruped "Puppy" and discuss the mechanisms of self-stabilization. Finally we discuss the relation of self-stabilization mechanisms and behavioral diversity.

\section{Behavioral Diversity of a Hopping Machine}

In this section we describe a new kind of hopping robot called Stumpy. Despite its simple structure, a salient feature of this robot is its large variety of behaviors ${ }^{1}$. In a set of systematic experiments, we will show how the behavioral diversity can be achieved by applying the principle of cheap design ${ }^{2}$.

\subsection{Design and Control}

Stumpy uses inverted pendulum dynamics to induce biped-like locomotion gaits. Its mechanical structure consists of a wide base in the form of a rigid inverted T-shape mounted on four compliant feet (Figure 1). An important feature of the base is its springy property. An upright "T" structure is connected to this base by a rotary joint labeled "waist". The horizontal beam of the upright " $T$ " is connected to the vertical beam by a second joint, a rotary joint that we call the

\footnotetext{
${ }^{1}$ The video clips are available at: http://www.ifi.unizh.ch/ailab/people/iida/stumpy/

${ }^{2}$ For further technical details, refer to the previous papers [19-21].
} 


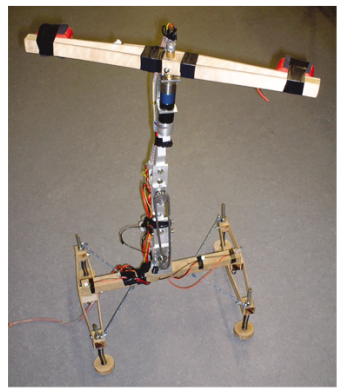

(a)

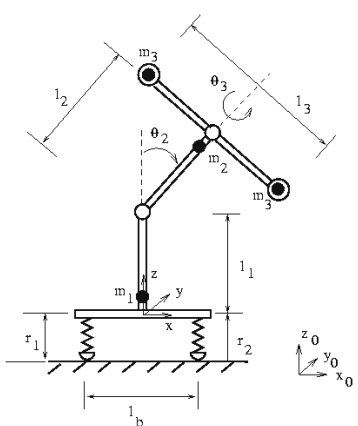

(b)

Fig. 1. The robot Stumpy. A photograph (a), and a schematic (b).

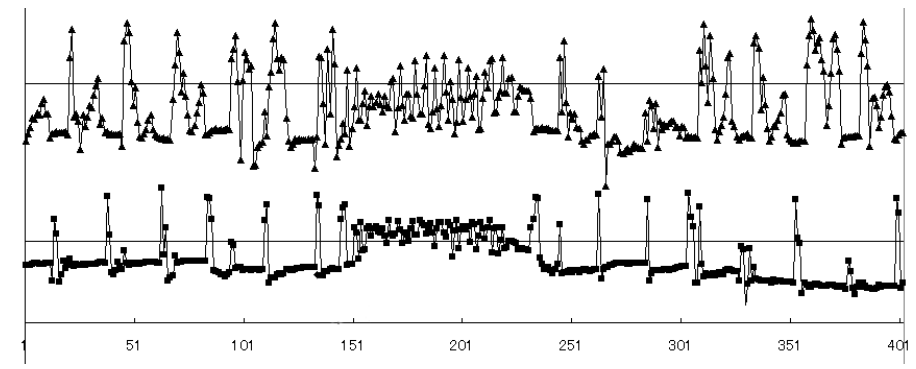

Fig. 2. Intrinsic stability of Stumpy. This graph shows the time series pressure data installed on the right and left feet (top and bottom graphs, respectively). The gait is disturbed by an external force around time step 150. A time step corresponds roughly to $1 / 20$ seconds.

"shoulder". By using this two degrees of freedom mechanical structure, the robot is able to perform many different locomotion behaviors including hopping and walking in a straight or curved line. Note that Stumpy does not have sensors to recognize its global states and so it does not know what behavior it is currently involved in. There is only local feedback that enables it to perform synchronized sinusoidal oscillations of the two joints.

\subsection{Intrinsic Stability and Behavioral Diversity}

In addition to the static stability which is achieved by a wide base and four feet, the dynamical stability is one of the major features in the behavior of Stumpy. Figure 2 shows the time-series pressure data measured at the feet of Stumpy. At around time step 150 an external force disturbance is exerted. The rhythmic pattern of the ground contact is generally retrieved after a certain period of chaotic behavior, in the figure after roughly 100 time steps. 


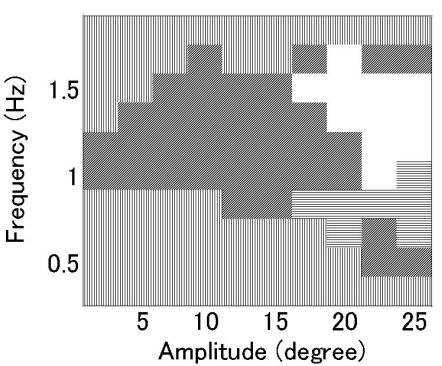

(a)

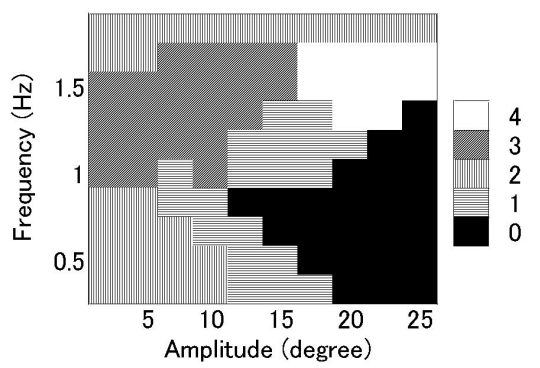

(b)

Fig. 3. Gait distribution diagrams. Gait distribution in Terrain 0 (a) and Terrain 1 (b). The shadings indicate the different gates: "4": Hopping, "3": Walking, "2": Shuffling, "1": Unstable, and "0":Fall.

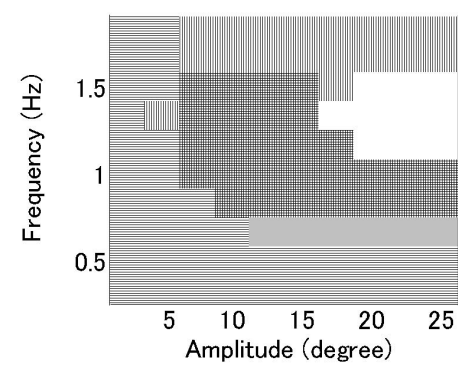

(a)

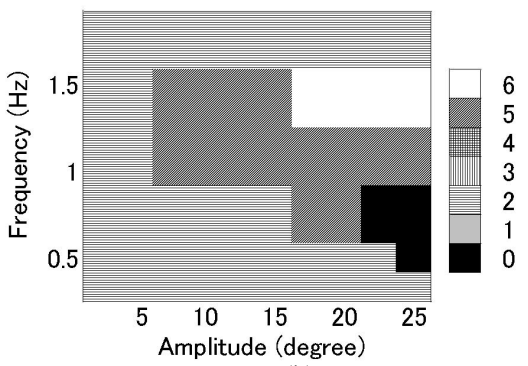

(b)

Fig. 4. Gait distribution diagrams of the lateral bounding experiments. Gait distribution on Terrain 0 (a) and Terrain 1 (b). The texture patterns indicate the different gaits: "6": Hopping to Right, "5": Hopping and Stay, "4": Hopping to Left, "3": Shuffling to Right, "2": Shuffling and Stay, "1": Shuffling to Left, and "0":Fall.

To explore this characteristic of self-stabilization further, we have conducted a systematic investigation in terms of the oscillation of the waist motor and the influence of environment. By simply changing two control parameters of the waist and the shoulder oscillation, frequency and amplitude, Stumpy exhibits a rich diversity of locomotion gaits. Figure 3 illustrates the variations of gaits when the amplitude and the frequency of the waist motor oscillation are varied in two different ground conditions (different coefficients of friction) labeled Terrain 1 and Terrain 2 . The gaits are categorized in terms of the time-series ground contact data, which indicate whether both feet are off the ground at a certain period in a gait cycle (Hopping), one foot is always on the ground (Walking), or both feet are always on the ground (Shuffling) (The "Unstable" gait means that there is no periodic pattern observed in the data). In the first experiment, we set the center of oscillation to be in the center with respect to the lower body, and we recorded the foot pressure data during 10 seconds of operation for 


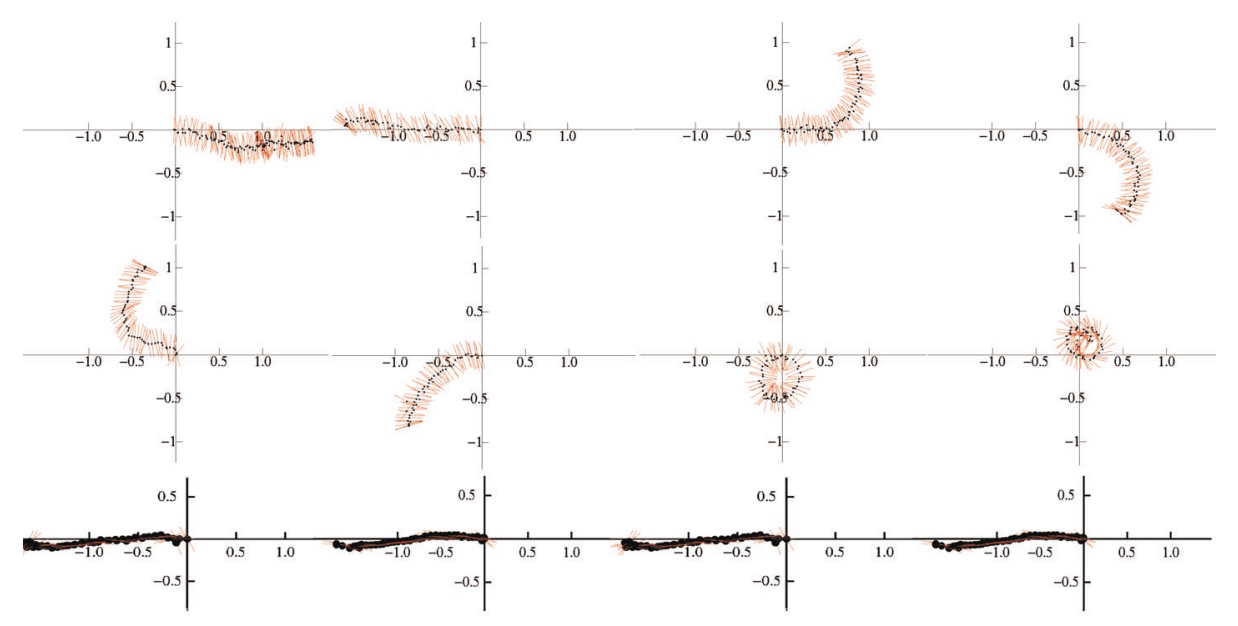

Fig. 5. Locomotion behaviors of Stumpy observed from top (The unit of these graphs is meters). Stumpy can control its movement direction, turning rate (Top and Middle panels), and lateral bounding (Bottom panels) by changing only a few control parameters. Black dots denote the trajectory of the body center, and the line illustrates the orientation of bottom base.

every parameter setting of frequency and amplitude. As a result, we observed four different gaits in this experiment. In a similar way, we also analyzed another kind of behavior called "lateral bounding". By setting the center of oscillation laterally to one side, Stumpy shows a locomotion behavior in the lateral direction. Compared to the previous experiment, the robot showed two basic gaits, i.e. Hopping and Shuffling, and the directionality of the movement depends on frequency and amplitude. Figure 4 shows the behaviors observed with respect to amplitude and frequency during the lateral bounding experiment.

So far, we used only the waist motor, but the behavioral diversity of Stumpy can be enhanced even further by adding another degree of freedom in the shoulder joint. By coupling the lateral and horizontal momentum induced by the rotary oscillation of the two motors, a hopping behavior can be achieved. While the waist oscillation generates a periodic hopping gait, the shoulder motor can control the horizontal forward/backward movement depending on the synchronization of these two oscillations. When the phase of two oscillations is reversed, the forward locomotion switches to backward. The turning rate can be controlled by biasing the speed of the horizontal oscillation: For example, faster rotation in the clock-wise direction leads to a turning movement (Figure 5).

The novelty of this kind of robot locomotion lies in its unique morphology. Because of the dynamic stability achieved by the wide springy base and the proper body design, many different patterns of physical interactions between the body, friction, actuation and control can be generated. 


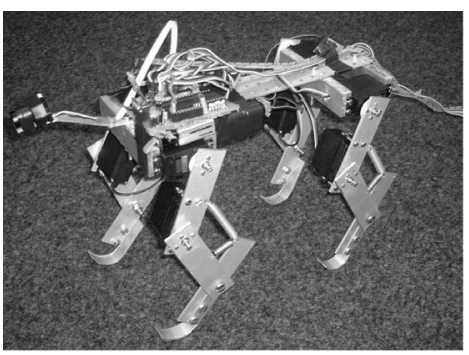

(a)

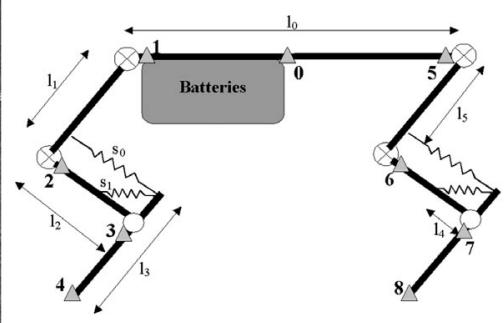

(b)

Fig. 6. The qudruped robot Puppy. A photograph of the quadruped robot (a), and schematic of the robot (b). The circles denote passive joints and the circles with a cross inside denote the joints controlled by servomotors. The triangles with numbers show the positions of LEDs which are used for visual tracking of the body geometry during locomotion experiments.

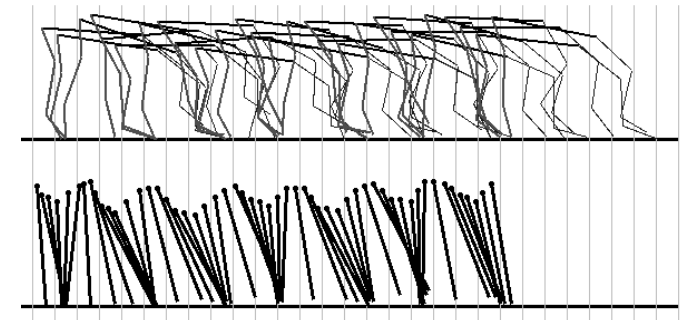

Fig. 7. Behavior analysis of a running experiment. The upper graph shows the behavior of the whole body based on the visual tracking of LEDs attached to the leg joints and the ground contacts. The lower graph shows the trajectory of a virtual linear hind leg.

\section{Self-Stabilization of Quadruped Running}

Cheap design is crucial for the rapid legged locomotion in order to increase the energy efficiency and reduce the computational cost. In this section, we describe a four-leg robot which exploits the elasticity of its components for running $^{3}$. The experimental results show that the running behavior is achieved by a self-stabilization mechanism, which can be used also for the control of forward velocity ${ }^{4}$.

\subsection{Design and Control}

Figure 6 shows the mechanical design of the running quadruped robot, Puppy, which is inspired by biomechanical studies. Each leg consists of two standard servomotors and one elastic passive joint in series, and the designs of all four

3 The video clips are available at: http://www.ifi.unizh.ch/ailab/people/iida/stumpy/

${ }^{4}$ Refer to [22] for more technical details. 


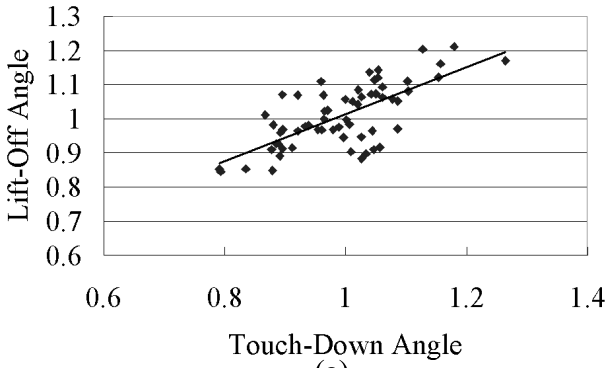

(a)

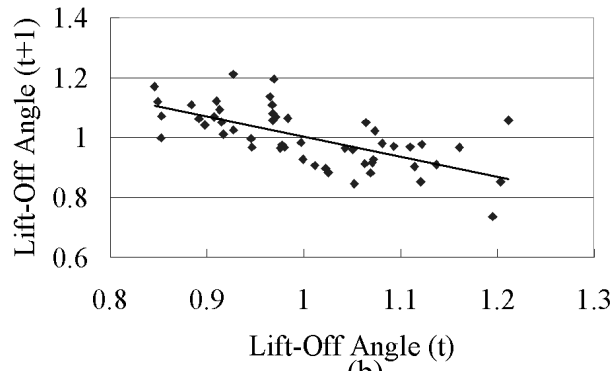

(b)

Fig. 8. Touch-down and lift-off angles of the qudruped Puppy. (a): The relation between touch-down and lift-off angles. The touch-down angles and lift-off angles are normalized by the corresponding mean touch-down and lift-off angles. (b): Relation between the normalized lift-off angles of successive leg steps.

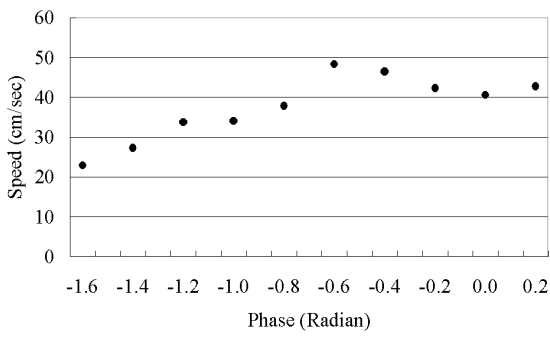

(a)

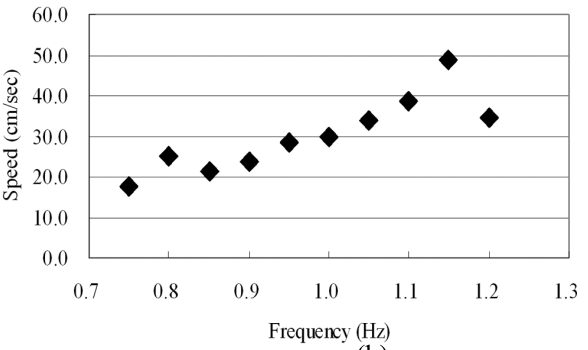

(b)

Fig. 9. . Speed diagrams of Puppy for different parameter values. Average speed vs, phase (a) and average speed vs. frequency (b) parameters.

legs are identical. This robot carries eight motors, batteries, and a micro-motor controller. To demonstrate a running gait, we apply a synchronized oscillation based control scheme to four motors in the hip and shoulder, where each motor oscillates through a simple sinusoidal position control. No sensory feedback is used for this controller except for the internal local feedback for the servomotors.

\subsection{Behavior Analysis of Self-Stabilization}

The behavior of the robot was extracted by a standard visual motion analysis, where the trajectories of the joints were visually tracked. Figure 7 shows a typical locomotion behavior extracted from a side view. The legs exhibit simple oscillations, but through the interplay of the elastic body structure, the mass distribution, the gravity and the ground friction, a natural quadrupedal running gait occurs, which includes periods in which all four legs are off the ground. In other words, there is a clear distinction between a stance and a flight phase. We found that this kind of running behavior significantly relies on the underlying self-stabilization mechanism. Although the control of the robot is extremely 
simple - the controller does not recognize the stance/flight phase, acceleration, or inclination - the robot maintains a stable periodic gait. This is due to the fact that it properly exploits its intrinsic body dynamics. The self-stabilization mechanism uses a unique characteristic of legs, which can be observed by the touch-down and lift-off angles of the virtual linear leg during the stance phase. Linear leg analysis means that the virtual line between the hip and the ground contact is estimated (Figure 7b). As shown in Figure 8b, the relation between successive lift-off angles is roughly linear. This means that, when a lift-off angle is lower, the subsequent lift-off angle is larger, and vice versa, which results in a stable touch-down angle over multiple leg steps. The underlying mechanism is implicitly contained in the entire body dynamics which has the effect that there is a linear relation between the touch-down and lift-off angles of the legs (Figure 8), which implies that a lower touch-down angle results in a larger lift-off angle, on average. The data shown in Figure 8 were collected from a series of 10 runs of 6 leg steps.

\subsection{Control of Forward Velocity}

Owing to the intrinsic self-stabilization property, the control of forward velocity can be easily realized by varying a single phase or frequency parameter of the oscillation. Figure 9 shows the average forward velocity with respect to the phase and frequency parameters of leg oscillation, which is extracted from the visual analysis explained above. It shows that, by simply varying the phase and frequency parameters, the velocity can be changed in the range from 20 to 50 $\mathrm{cm} / \mathrm{sec}$, approximately. It is interesting to note that this control strategy of the forward velocity by means of the oscillation phase and frequency is one of the simplest possible control parameters because it can be a simple time delay in the neural substrate.

\section{Discussion and Conclusion}

Exploiting self-stabilization mechanisms seems to be a common strategy for legged animals in nature and some of them have been explored in biology and robotics (e.g. [14-16, 18, 23, 24]). However, the case studies shown in the previous sections provide further interesting aspects for a comprehensive understanding of embodied adaptive locomotion.

\section{Self-Stabilization and Behavioral Diversity}

The simplicity of the control of Stumpy and Puppy is mostly due to the selfstabilization mechanisms. Without sensory feedback, the locomotion processes are maintained by properly exploiting the interaction of body material and dynamics (e.g. aluminium, springs, and mass distribution), environmental (e.g. friction and rough terrain), and control (e.g. amplitude and frequency). Moreover, as illustrated in these case studies, the self-stabilization mechanisms not 
only simplify the control, but also significantly influence the locomotion function itself. In other words, sophisticated design and control is not always required in order to achieve rich behavioral diversity. The lateral bounding behavior of Stumpy provides a good example of how behavioral diversity can be achieved by exploiting the body dynamics. By simply changing the oscillation frequency, Stumpy exhibits very different behaviors in terms of gait and direction. As another example, the control of Puppy's forward velocity is no longer possible by just varying the rotation speed of the motors, but the control parameters, phase and frequency, have to be varied in order to influence the body dynamics.

An interesting feature of the proposed approach is the fact that there are a few different control parameters instead of only one parameter, which can be used for the same purposes. For example, as shown in Figure 3, the locomotion gait of Stumpy can be controlled by both amplitude and frequency, and the same holds for the direction and the gait of lateral bounding locomotion. The forward velocity of Puppy also illustrates this point clearly, i.e. both parameters, phase and frequency, are able to control the velocity. Note that these control parameters are not controlling the locomotion function directly, but indirectly by changing the dynamics.

In the locomotion experiments shown in this paper, Stumpy and Puppy were operated mostly at the resonance frequencies of the systems in order to exploit the body dynamics. However, it is clear that the other kinds of physical interactions which influence the body dynamics should be considered as well. Not only simple linear springs and rigid materials, but properties such as damping and mass distribution need to be explored to better understand how behavioral diversity can be achieved. In addition, it should also be mentioned that the behavioral diversity could be potentially enhanced further by operating the system at non-resonance frequencies for more torque demanding stationary tasks. These two strategies of behavior control need to be explored further as well.

\section{Toward Embodied Adaptive Locomotion}

Although we have focused on the functional aspects of locomotion in this paper, this approach provides additional insight into embodied adaptive behavior or intelligence in general. The control of behavior is quite often the major research interest of adaptive locomotion, but the use of body dynamics is also a fundamental mechanism to properly understand behavioral diversity. As illustrated in the case studies of this paper, the functions of the system are no longer separable from the constraints derived from embodiment, if the behavior of the robots highly depends on its body dynamics: there is no longer a clear separation of hardware and control. In this sense, locomotion behavior is also essential for high-level cognition, as it enables the agent to construct a "body image" that on the one hand can be used to guide behavior in the real world and on the other as a basis for metaphors on top of which something like cognition can be bootstrapped. 


\section{Acknowledgement}

We would like to thank Raja Dravid and Chandana Paul for the productive discussion and collaboration. This work is supported by the Swiss National Foundation, grant No. 20-68198.02.

\section{References}

1. Brooks, R. A.: A robot that walks: emergent behaviors from a carefully evolved network. Neural Computation 1(2) (1989) 253-262

2. Vukobratovic, M., and Stepanenko, J.: On the stability of anthropomorphic systems. Mathematical Biosciences 15 (1972) 1-37

3. Yamaguchi, J., Soga, E., Inoue, S. and Takanishi, A.: Development of a bipedal humanoid robot - control method of whole body cooperative dynamic biped walking. In Proc. IEEE Int. Conference on Robotics and Automation (1999) 368-374

4. Hirose, M. Haikawa, Y., Takenaka, T., and Hirai, K.: Development of humanoid robot ASIMO. Proc. Int. Conference on Intelligent Robots and Systems (2001)

5. Loeffler, K., Gienger, M., Pfeiffer, F.: Sensor and control design of a dynamically stable biped robot. ICRA 2003 (2003) 484-490

6. Arikawa, K., Hirose, S. Development of quadruped walking robot TITAN-VIII, Proceedings of International Conference on Intelligent Robots and Systems (IROS96) (1996) 208-214,

7. Collins, S. H., Wisse, M., and Ruina, A.: A three-dimentional passive-dynamic walking robot with two legs and knees. International Journal of Robotics Research 20 (2001) 607-615

8. McGeer, T.: Passive dynamic walking. International Journal of Robotics Research 9 (1990) 62-82

9. Wisse, M. and van Frankenhuyzen, J.: Design and construction of MIKE: A 2D autonomous biped based on passive dynamic walking. Proceedings of International Symposium of Adaptive Motion and Animals and Machines (AMAM03) (2003)

10. Pfeifer, R. and Scheier, C.: Understanding Intelligence. The MIT Press, (1999)

11. Taga, G., Yamaguchi, Y., and Shimizu, H.: Self-organized control of bipedal locomotion by neural oscillators in unpredictable environment. Biological Cybernetics 65 (1991) 147-159

12. Fukuoka, Y., Kimura, H., and Cohen, A. H.: Adaptive dynamic walking of a quadruped robot on irregular terrain based on biological concepts, Int. Journal of Robotics Research, Vol.22, No.3-4, (2003) 187-202

13. Ishiguro, A., Ishimaru, K., Hayakawa, K., and Kawakatsu, T.: Toward a "wellbalanced" design: A robotic case study -How should control and body dynamics be coupled?-, in Proc. of The 2nd International Symposium on Adaptive Motion of Animal and Machines. (2003)

14. Kubow, T. M., Full, R. J.: The role of the mechanical system in control: a hypothesis of self-stabilization in hexapedal runners. Phil. Trans. R. Soc. Lond. B 354 (1999) 849-861

15. Raibert, H. M.: Legged robots that balance. The MIT Press (1986)

16. Buehler, M.: Dynamic locomotion with one, four and six-legged robots. Journal of the Robotics Society of Japan 20(3) (2002) 15-20

17. Alexander, R. McN.: Three uses for springs in legged locomotion, The International Journal of Robotic Research, 9, No. 2 (1990) 53-61 
18. Seyfarth, A., Geyer, H., Guether, M., and Blickhan, R.: A movement criterion for running. J. Biomech. 35(5) (2002) 649-655

19. Iida, F.,: Exploiting friction for a hopping robot. Proc. of Adaptive Motion of Animals and Machines (2003)

20. Iida, F., Dravid, R., Paul. C.: Design and control of a pendulum driven hopping robot. Proceedings of International Conference on Intelligent Robots and Systems 2002 (IROS 02) (2002) 2141-2146

21. Paul, C., Dravid, R., Iida, F.: Control of lateral bounding for a pendulum driven hopping robot. Proc. of 5th International Conference on Climbing and Waling Robots (CLAWAR 2002) (2002) 333-340

22. Iida, F., and Pfeifer, R.: "Ceap" rapid locomotion of a quadruped robot: Selfstabilization of bounding gait. Proc. of Intelligent Autonomous Systems 8, Groen, F. et al. (Eds.), IOS Press (2003) 642-649

23. Cruse, H., Bartling, C. H., Brunn, D. E., Dean, J., Dreifert, M., Kindermann, T., and Schmitz, J.: Walking: A complex behavior controlled by simple systems. Adaptive Behavior 3(4) (1995) 385-418

24. Herr, H. M., McMahon, T. A.: A trotting horse model, The International Journal of Robotics Research, 19, No. 6 (2000) 566-581 